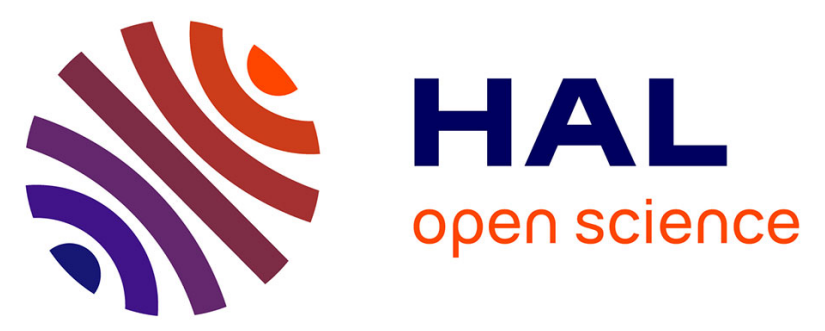

\title{
Using Sensory Substitution of Median Sensory Deficits in the Traumatized Hand to Develop an Innovative Home-Based Hand Rehabilitation System
}

Alessandro Semere, Yohan Payan, Francis Cannard, Bruno Diot, Nicolas Vuillerme

\section{To cite this version:}

Alessandro Semere, Yohan Payan, Francis Cannard, Bruno Diot, Nicolas Vuillerme. Using Sensory Substitution of Median Sensory Deficits in the Traumatized Hand to Develop an Innovative HomeBased Hand Rehabilitation System. 13th International Conference On Smart homes and health Telematics (ICOST'2015), Jun 2015, Genova, Switzerland. pp.53-63, 10.1007/978-3-319-19312-0_5 . hal01217801

\section{HAL Id: hal-01217801 \\ https://hal.science/hal-01217801}

Submitted on 20 Oct 2015

HAL is a multi-disciplinary open access archive for the deposit and dissemination of scientific research documents, whether they are published or not. The documents may come from teaching and research institutions in France or abroad, or from public or private research centers.
L'archive ouverte pluridisciplinaire $\mathbf{H A L}$, est destinée au dépôt et à la diffusion de documents scientifiques de niveau recherche, publiés ou non, émanant des établissements d'enseignement et de recherche français ou étrangers, des laboratoires publics ou privés. 


\title{
Using sensory substitution of median sensory deficits in the traumatized hand to develop an innovative home- based hand rehabilitation system
}

\author{
Alessandro Semere ${ }^{1,2}$, Yohan Payan $^{3}$, Francis Cannard ${ }^{4}$, Bruno Diot ${ }^{2,5}$, Nicolas \\ Vuillerme $e^{2,6}$ \\ ${ }^{1}$ Grenoble University Hospital, Plastic Hand and Burns Surgery, France \\ \{a.semere\}@chu-grenoble.fr \\ ${ }^{2}$ Univ. Grenoble-Alpes, AGIM, France \\ \{alessandro.semere, nicolas.vuillerme\}@ agim.eu \\ ${ }^{3}$ Univ. Grenoble Alpes, TIMC-IMAG, F-38000 Grenoble, France \\ CNRS, TIMC-IMAG, F-38000 Grenoble, France \\ \{yohan.payan\}@imag.fr \\ ${ }^{4}$ Texisense, Montceau-les-Mines, France \\ \{francis.cannard $\} @$ texisense.com \\ ${ }^{5}$ IDS, Montceau-les-Mines, France \\ \{b.diot\}@ids-assistance.com \\ ${ }^{6}$ Institut Universitaire de France, Paris, France
}

\begin{abstract}
Post-traumatic median nerve sensitive deficits are frequent. They are a source of permanent handicap that dramatically decreases the level of autonomy and the quality of life of persons suffering from these deficits. Surgical repair is possible, but the results are not always functionally useful. Therefore, prosthetic approaches do represent an alternative solution that needs to be explored. Along these lines, this paper describes an innovative homebased hand rehabilitation system device that exploits sensory substitution of median sensory deficits in the traumatized hand. It is composed of a glove bearing smart textile pressure sensors and a wristband providing vibratory biofeedback to the user. The goal of this sensory-substitution system is to provide for patients an effective method to compensate the lack of sensitivity of the finger pads and to recover a functional hand use. This innovative system is intended to be employed for assessment, training and rehabilitation exercises at home.
\end{abstract}

Keywords. home rehabilitation, sensory substitution, hand trauma, median nerve

\section{Introduction}

Hand traumas strike 1,400,000 patients every year in France; 620,000 of them lead to severe injuries. They are essentially caused by everyday life, occupational, and road accidents [1]. In the severe cases, sequelae are frequent. This has an important 
socio-economical impact: according to the FESUM (Fédération Européenne des Services d'Urgence de la Main - European Federation of Hand Emergency Services), $1 / 3$ of the occupational accidents involve the hand, $1 / 4$ of the lost days of work is because of these traumas, $1 / 10$ of the accidents leads to a PPD (Permanent Partial Disability, which is an index rating the permanent handicap resulting of a trauma, after consolidation) and 1/3 of the PPD is the result of hand accidents. On average, a hand accident with a work stoppage costs from 1,000 to 2,000 euro: $80 \%$ of this amount is represented by indemnities (half by work stoppages, half by IPPs). This handicap is more important as the lesions impair fine hand use of manual workers. Hand motricity can be restored thanks to palliative interventions: nerve grafts, neurotizations and tendon transfers. Innervated digital flaps can locally restore sensitivity, but the donor site is then shorn of its function. Finally, in case of pluridigital deficit, there is no complete mean of substitution. Multi-daily sensory rehabilitation can help to recover useful (although uneven) sensitivity, but needs motivation, discipline, time and patience. Unfortunately, access to daily training with a specialist is not always possible. The lack of time of the patients or their living distance from the physiotherapy office are also obstacles to the rehabilitation. Finally, the cost of the care can quickly become exorbitant.

The work of Bach-y-Rita on sensory substitution for the blind [2] as well as the more recent scientific works on the Tongue Display Unit (TDU) for pressure ulcer prevention [3], balance problems [4] and the guiding of surgical gesture [5] have inspired the conception of an innovative device enabling to rehabilitate or to palliate these sensory deficits. Following this approach, this paper describes an innovative home-based hand rehabilitation system device that exploits sensory substitution of median sensory deficits in the traumatized hand.

\section{Materials and methods}

The device has been designed according to the organic facts and psychomotor theories on haptics. Its actual development was possible by the manufacture of smart textile pressure sensors [6].

\subsection{Physiopathology of hand nervous injuries}

The hand is a complex organ. Its gripping functions are possible thanks to a perfect biomechanical structure made of bones, joints, ligaments and tendons. A two-way circuit controls these motor skills: the efferent nerve fibers command muscle contractions (motricity), the afferent nerve fibers give the central nervous system (CNS) a feedback of the force of these contractions and the actual localization of the fingers and joints in space (sensitivity), and the CNS synthesizes the inputs and outputs, coordinating the movements (integration). The three nerves carrying the efferent and afferent fibers are the median nerve, the ulnar nerve, and the radial nerve. Each of them has a different motor function and sensitive territory. The median nerve is functionally the most important to perform the bidigital grip: it innervates the flexor 
muscles of the fingers and the abductor muscles of the thumb, and its sensitive area covers the palmar side of the first, second, third, and radial side of the fourth finger. The sensitive and motor fibers circulate in mixed nerves. This implies that in case of complete section of a peripheral nerve, both efferent and afferent paths are interrupted. The treatment is nerve suture under optical magnifying, to restore the continuity of its channels and to guide the axonal regrowth. Nevertheless, the recovery is long and uneven. While motor skills often return to their previous level (or with a force limitation of $25 \%$ to $50 \%$, but still functional), sensitivity is more problematic [7, 8]. Recent works specifically implicate sensitive deficits in the troubles of the function of the thumb-index grip [9]. They suggest that a loss of sensitivity leads to an alteration of the coordination of fine gestures. Actually, we can observe during prehension a degraded control of the values of the pad pressure because of the lack of feedback, which leads to an increase of the security margins [9-16], as well as an increase of the variations of these pressures of $11 \%-12 \%$ (vs $5 \%-6 \%$ for a healthy individual [10]). This implies that for light charges, objects are involuntarily dropped $[10,14]$. Moreover, the displacement of the center of pressure of digital pads during fine pinch is multiplied by 5 in sensitivity troubles [10], leading to an increase of the tangential forces followed by a rotation of the object. The patient responds inappropriately by increasing his security margin, which more often can lead to the dropping of the object $[10,12]$. These troubles are independent of the maximal grip force of the patient, which is reduced by $25 \%$ to $35 \%[9,10]$. Indeed, the security margins are studied only during the manipulation of light objects (less than $500 \mathrm{~g}$ ), needing a force of $10 \mathrm{~N}$ for a maximal force of $50 \mathrm{~N}$ [10]. Finally, we can note that anticipation of the movement and visual control during its execution tend to normalize these results $[14-15,17]$, compensating the lack of touch.

\subsection{Sensory substitution}

Sensory substitution is a concept introduced in the sixties by Professor Paul Bachy-Rita [18]. It consists of transforming the characteristics of one sensory modality into stimuli of another sensory modality. This has been used to restore the ability of people to perceive a certain defective sensory modality by using sensory information from a functioning sensory modality. In general, a sensory substitution device consists of one or several sensors, a coupling system and one or several stimulators. The sensor records stimuli and gives them to a coupling system that interprets these signals and transmits them to a stimulator. As stated above, the median nerve is the most important nerve implied in the fine grip, its injuries are fairly frequent, and its recovery is often poor. These facts and the literature $[19,20]$ led us to think that this concept of sensory substitution could be used to substitute the sensitivity of the median nerve by an artificial system, in order to improve the control of fine grip. The aim was to develop a device capable of transducing the sense of touch of this deficient area through another modality (true sensory substitution), or with the same modality (i.e. touch) but on another healthy localization. This needs a study of the sense of touch, together with a state of the art as concerns the existing artificial input and output systems. 
There are 17000 receptors in the glabrous hand, to sense the different types of stimuli: exteroceptive (pressure, stretching and vibrations), proprioceptive (positioning of joints in space), and thermoalgic (temperature and pain). Their stimulation is the first step of the afferent path. There are four basic types of exteroceptive receptors: Pacinian corpuscle, Meissner corpuscle, Ruffini endings and Merkel nerve endings. Each of them has a different response latency to stimuli (fast adaptive fibers FA or slow adaptive fibers SA), a variable pressure threshold, and various numbers of sensitive endings and depth under the skin [21]. This makes them more or less sensitive to pressure discrimination, spatial discrimination (two-point static detection), temporal discrimination (vibration), and stretching. The complexity of this system is high; it is therefore difficult to simulate precisely all of these criteria. Moreover, this is not necessarily suitable: indeed, an excessively exhaustive input information would proportionally complicate the output information. The result would be an increase of the cognitive load, hence a decrease of the accuracy and the speed of interpretation of the signal (which is exactly the opposite we are trying to achieve). For the development of the device, we have decided that our sensors will detect only useful pressure changes with a useful spatial discrimination. This feedback is essential for grip control and shape recognition. We finally dropped the detection of stretching and vibration. The sensitivity of a hand to pressures is high. In clinical routine, it is measured with Semmes-Weinstein monofilaments (SWM), which are semi-rigid nylon threads, calibrated to bend at a precise force [22]. A normal hand has a minimal sensitivity of 0.008 gf to 0.07 gf (grams-force, 1 $\mathrm{gf}=9.80665 \mathrm{mN}$ ), with a spatial discrimination of $2 \mathrm{~mm}$ to $6 \mathrm{~mm}$. Finally, the high sensitivity of the fingertips is not always needed. Indeed, some zones are functionally more important than others. The grips mostly used are the key grip (pad of the thumb and of the radial side of the index), the needle-thread grip (tip of the thumb and of the radial side of the index), the tridigital grip (thumb, index and radial side of the middle finger), the button push (index tip and pad) and the lighter (pad and tip of the thumb).

To supply a sensory feedback, all of the five senses are theoretically useful and usable. However, for our application, we needed something light and easily bearable all day long in everyday life. Sight is naturally complementary to touch, even in healthy people [23]. The evaluation of shape, volume and size of objects is done by eye control thanks to its wide receptive field, while textures are discriminated essentially by exploratory touch thanks to high vibrotactile accuracy. However, the only visual feedback about grip forces is given by the deformation or the slipping of the lifted objects, respectively translating the application of an excessive or an insufficient grip force, and leading to the breaking by fall or burst of non-deformable or fragile objects. We have decided not to implement a visual control, since it would overload this channel already sufficiently sought. Moreover, the permanent overlooking of a screen didn't seem appropriate for use in daily life. Hearing is a fast and precise modality of feedback, with great discriminatory amplitude, but we did not think that the permanent wearing of a headset would be comfortable for current use. Taste and smell are too slow, too imprecise and too variable according to physiological factors and seemed technically difficult to implement in our study. Touch was the first kind of feedback to be invented, and the simpler to implement. Moreover, simple and portable devices can be easily manufactured. We hence have decided to use this modality in our device. 
Finally, touch can be stimulated by different kinds of devices [20]. Low frequency, low amplitude mechanical deformation, raising bumps against the skin and rendering a relief that can be explored by touch. One can distinguish between continuous contact with an object, and "make-and-break" contact, in which an object is brought in and out of contact with the body. The skin possesses an especially high sensitivity to the latter. For the same reason, Vibrotactile stimulations are also easily recognized, especially if frequencies are chosen to maximize Pacinian FA II receptor sensitivity (highest near $250 \mathrm{~Hz}$ ), and may be effectively transmitted through an air gap. Electrotactile stimulation excites the afferent nerves directly rather than the tactile receptors themselves, via electrodes of different types or by fine wires inserted into the skin. Various afferent types can be excited differentially through the design of the drive signal and electrical contacts. Force feedback is by nature meant to access primarily the kinesthetic haptic channel. However, when force feedback devices interact with the cutaneous tactile sense, friction phenomena, vibration, or contact transients are inevitably generated. We found thermal and air or liquid jets displays too slow or complicated to be implemented in this project.

We decided to develop a vibrotactile device, acting as a low frequency mechanical stimulator. In other words, the various forces sensed would be coded by variable bursts (in duration and/or in frequency) of constant high frequency vibrations.

\section{Results}

The device presented here is composed of three distinct elements:

(1) a glove (bearing smart textile pressure sensors),

(2) a software (receiving the data form the sensors, treating and recording the signal), and

(3) a wristband, providing vibrotactile feedback.

When the user grasps an object with his fingers, the wristband vibrates according to the pressure measured by the sensors. The sensory-impaired patient would have the possibility to use vibrotactile biofeedback to control his grip and to maintain an appropriate force to avoid the involuntary dropping of objects.

The glove (Texisense ${ }^{\circledR}$ ) bears eight textile polyamide (nylon) sensors, thin-film coated with a piezo-resistive polymer (Fig. 1a). Each sensor is circular and has a 1 $\mathrm{cm}$ diameter. These sensors are fixed to the glove with glue and threads. They are placed on the three first fingers, four on the tip and pad on radial and ulnar sides of the thumb and two on the tip and pad on radial side of the index and middle finger. Each of them is connected to two conductive threads coated with silver. The threads run across the dorsal side of the glove, and are connected to metal buttons placed on the back of the wrist. Then, sixteen regular soft wires connect the glove to the acquisition circuit, powered by a 32 bit microcontroller unit (STM-32 Cortex from ST Microelectronics), which can be connected to a computer by a regular mini-USB to USB connection.

ST Microelectronics has provided the drivers for Microsoft Windows, which create and manage a virtual COM port between the glove and the computer. The signal acquisition and treatment software (Fig. 1b) has been written in $\mathrm{C}++$ using 
.NET framework 3.5, with Microsoft Visual Studio 2008. It offers several basic functions to exploit the capabilities of the device. The acquisition circuit provides a 64-bit signal ( 8 bit from each sensor) that is recovered from a specific memory address, at a $10 \mathrm{~Hz}$ sampling rate. A simple mean 10-values filter has been implemented. The values of the sensors can be displayed in raw state or converted to Newtons $(\mathrm{N})$ or to grams-force. The values for each sensor are displayed in real time in eight textboxes, as well as their minima and maxima. They are also visualized on dynamic .NET windows forms bars. Finally, a color graph (realized using the open source zedGraph library [24]) shows the curves of evolution of these values compared to the maxima. The values can be stored to a .txt file in real time, raw or converted. Finally, to keep the useful values to control the feedback only, two operations are successively executed: (1) a threshold of $278 \mathrm{gf}$ is applied to each value (each sensor exceeding the threshold being considered as "activated"), and (2) the mean value of the "activated" sensors is calculated.

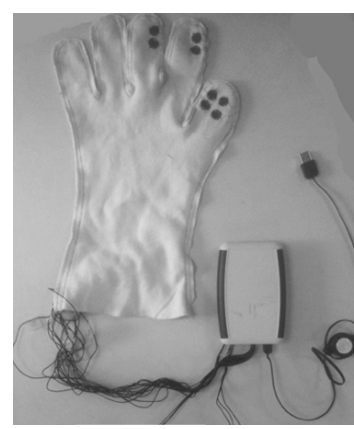

(a)

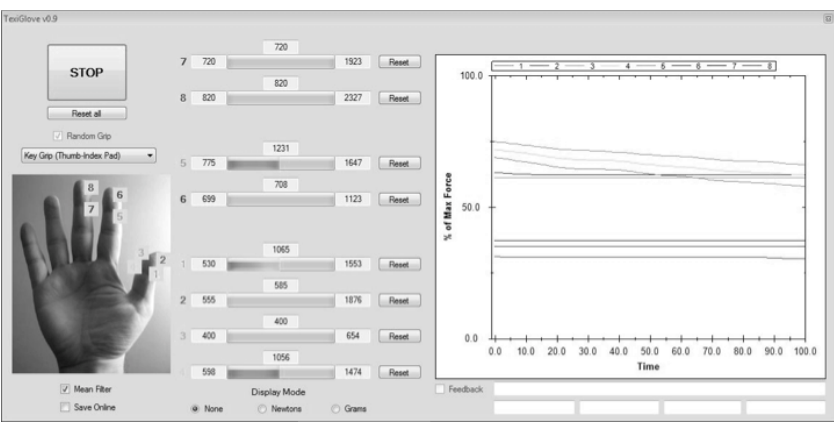

(b)

Fig. 1. The glove (a) and the signal acquisition and treatment software (b)

The feedback system is composed of a rubber and plastic wristband, containing an eccentric-mass brush motor and an USB motor controller. A Bluetooth vibrating wristband for mobile phones (MB20, Movon®) has been disassembled to keep only the motor $(1.3 \mathrm{~V}, 70 \mathrm{~mA}, 9000-12000 \mathrm{rpm})$, and a serial resistor (100 Ohm, $1 \%$ tolerance) has been added. The power is provided by a $6 \mathrm{~V}$ external stabilized transformer, via a modified USB cable. The control unit is an USB commercial motor controller (PhidgetMotorControl LV 1060, Phidgets®), powered by USB, and able to control the acceleration and velocity of the low-voltage brush motor thanks to the provided $\mathrm{C}++$ librairies. Bursts of vibrating impulses are generated to provide vibrotactile feedback. The vibrotactile stimulation is absent if the mean value of the activated sensors is lower than $278 \mathrm{gf}$, becomes continuous if between $278 \mathrm{gf}$ and 555 gf, then alternating gradually slower with the increase of the pressure. Over $1390 \mathrm{gf}$, the frequency of alternation is slow and constant.

We calibrated the sensors with an electronic precision scale (Mettler PE 1600, Max $1600 \mathrm{~g}$, Min $5 \mathrm{~g}, \mathrm{e}=0.1 \mathrm{~g}, \mathrm{dd}=0.01 \mathrm{~g}$ ), a laboratory articulated chassis, with an infra-millimetric manual movement control, and a metal cylindrical piece (diameter $=$ $8 \mathrm{~mm}$ ), isolated at its tip by a rubber band. Each sensor has been put on the balance 
and under the cylindrical piece. A piece of wood has been put under and inside the glove. This avoids short-circuits between the sensors, the threads and the balance board. After putting the balance to zero, the cylindrical part was progressively lowered, until the desired force was obtained. These forces have been obtained by the conversion of the forces $\mathrm{F}$ (in gf) given by $\mathrm{SWM}$ in pressures $\mathrm{P}(\mathrm{Pa})$, by the formula $\mathrm{P}=\mathrm{F} / \mathrm{S}$, where $\mathrm{S}$ is the surface of the section of the SWM given by the literature [25]. Then, each pressure value has been converted to a force value considering the section of the $8 \mathrm{~mm}$ diameter cylindrical metal piece. Finally, the raw value displayed by the software was then reported in a table. The raw curves have been drawn, and the equations of mean root square deviation lines have been calculated (Fig. 2). The latter are used by the software to convert the raw values to grams-force and to Newtons. The evolution after constant stimulation ( $278 \mathrm{gf}$ ) has been measured, to look for the implication of this mixed effect in time (Fig. 3). After one minute, the evolution is negligible (Maximum $=2.7 \%$ ).

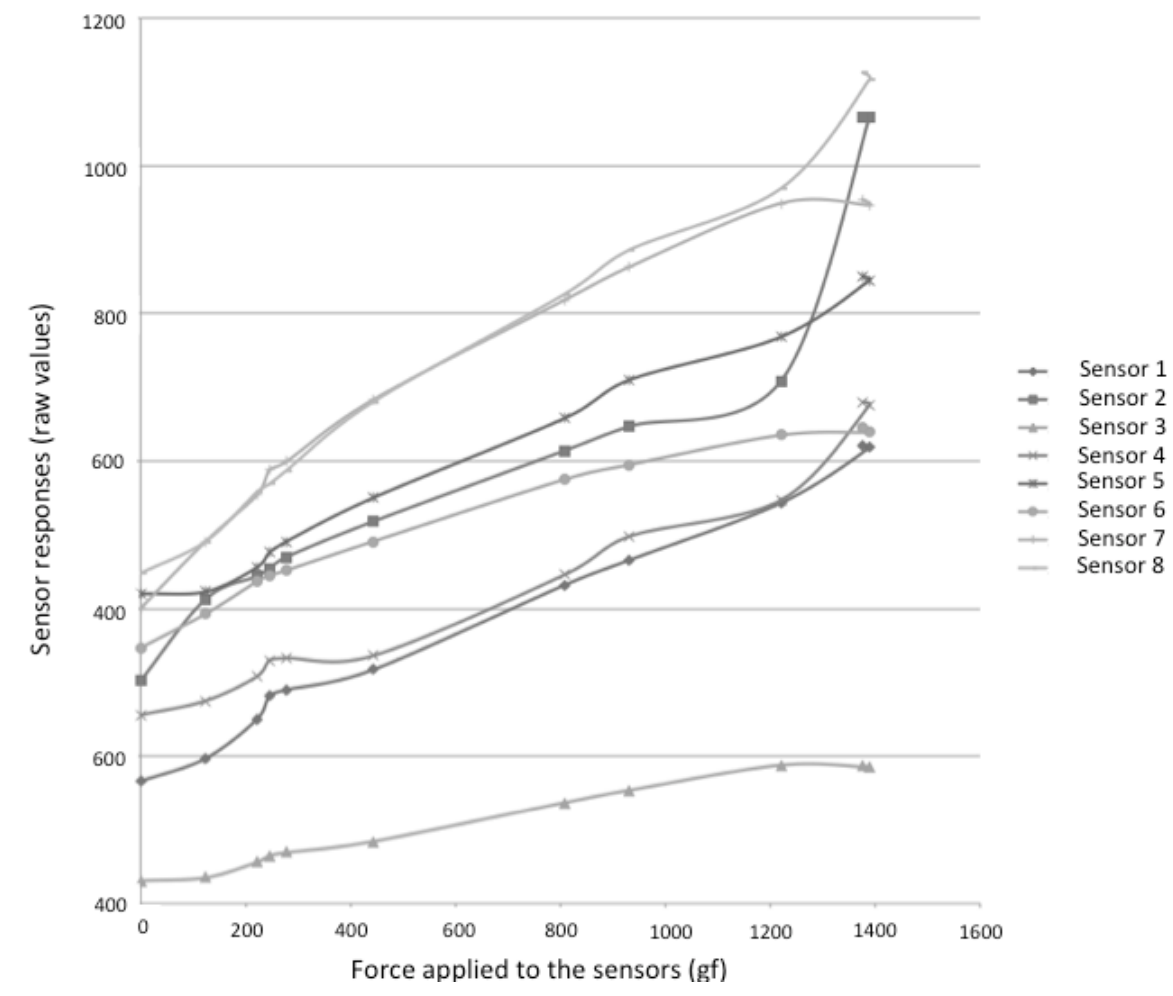

Fig. 2. Sensor responses to increasing applied pressures. 


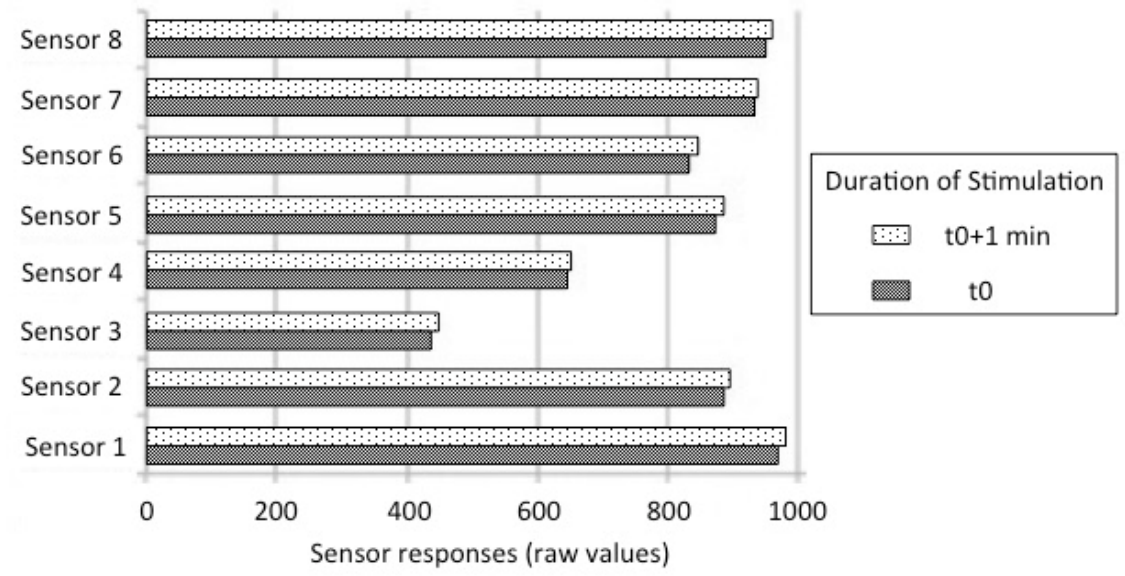

Fig. 3. Sensor responses after constant stimulation to $278 \mathrm{gf}$.

\section{Discussion}

The presented work described an innovative home-based hand rehabilitation system device. It is composed of a glove bearing smart textile pressure sensors and a wristband providing vibratory biofeedback to the user that could be employed for training and rehabilitation exercises at home. This device exploits the concept of sensory substitution of median sensory deficits in the traumatized hand. It is designed to provide for patients an effective method to rehabilitate or to compensate the lack of sensitivity of the finger pads and to recover a functional hand use.

By showing that this device is actually able to sense a pressure and to send a vibrotactile feedback to the user, our results are encouraging and promising. However, because of several issues encountered during the development, several improvements still have to be introduced.

Indeed, while at first sight we could observe a linear relationship between forces and values returned, a more accurate analysis could have shown some unwanted fluctuations.

- Mixed piezo-resistive effect: The sensors are made of fine knit polyamide fibers coated with a piezo-resistive polymer. Like any other piezo-resistive device, the resistance of the sensors decreases with the mechanical stress. This works on the principle of the tension divider bridge. However, these fibers have a far more complex behavior. The force applied on them is distributed considering their geometry, their type of knit, the tightening... The fibers being conductive and deformable, the squeezing increases the surface contact and decreases the resistance independently from the piezo-resistive effect. This mixture of effects implicates that the response of the sensors can only be approached by calibration. Another observation is that the raw value of the sensors, once stimulated and released, drops under the baseline level and progressively rises to 
return to its starting point. This is probably due to the hysteresis of the fibers, behaving like an elastic body.

- Manufacture problems: Each sensor is handmade so individual behavior is rather unpredictable. Moreover, the sensitivity seems to vary with the amount of glue used in the manufacturing. They are also fixed with a different amount of turns of conductive threads. This explains their various profiles of response, and the need for an individual calibration. Also, button connectors are loosely fixed to the glove and their movements and short-circuits cause variations and sudden drops of the sensed values. They tend to decrease the repeatability of the measurements. The measure of the various sensors has been done several times with no load. The result was variable, and some sensors have a more reliable response than others (Fig. 4a). The best sensor has a standard deviation of 6.8 $(\mathrm{M}=871,6$, less than $1 \%)$ and the worst $56.3(\mathrm{M}=553,9$, over $10 \%)$. This has been repeated with a charge of 278 gf with similar results (Fig 4b).

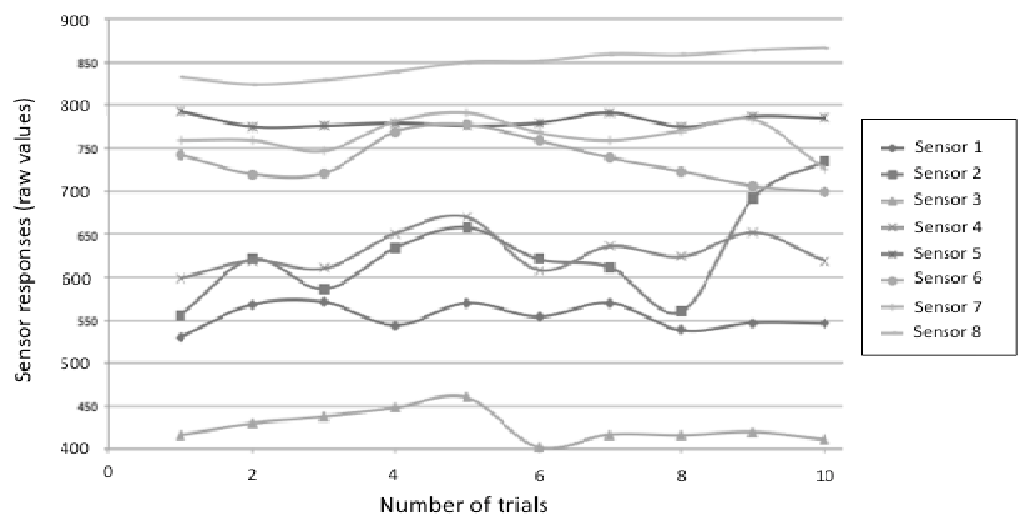

(a)

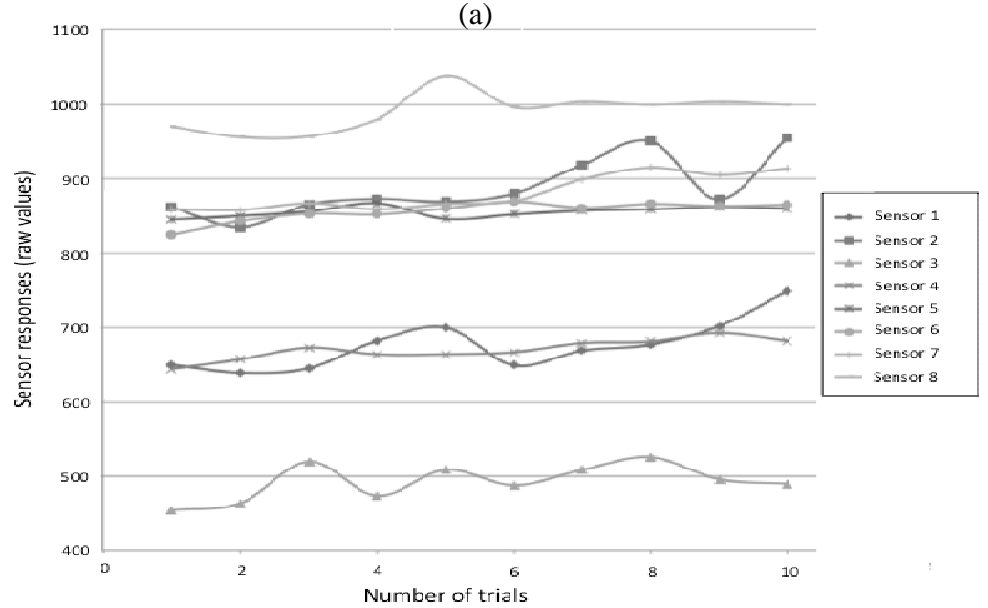

(b)

Fig. 4. Repeatability of the measurements with no load (a) and with 278gf (b). 
- Calibration method: The precision of the setup for calibration could be questionable. While the pressure measured is digitally precise, the application of the force is not. With the above setup, the pressure applied on the sensors is manually modified, and the observer reads and writes down the value. There is no digital synchronization between the device and the calibration system.

Finally, the feedback system has been manufactured with simple and low-cost materials for rapid proof-of-concept purposes. The stimulator needed a minimal discrimination level, to make the difference between "contact" and "no contact". The threshold level has been put to 278 gf, which corresponds to the lower precision of force detection of a normal hand (reported to a $8 \mathrm{~mm}$ diameter section). The decision has been taken to have the better compromise between noise and useful signal, but no systematic assessment has been done. The evaluation of the most efficient vibrotactile pattern for discrimination is included in our immediate plans.

\section{Conclusion}

Home-based rehabilitation is increasingly used to improve compliance program and to reduce health-care costs. Along these, we have presented here innovative vibrotactile sensory substitution in hand sensitive deficits for hand home-based rehabilitation. Although some technical aspects (e.g., calibration of the sensors) still need to be further studied and developed, these preliminary results are very encouraging. The ultimate aim of this device is to empower the patients in their own rehabilitation, allowing them to become the major players of their healing. They could plan the training sessions at home according to their time schedule and adjust their exercises according to their pain and tireness, without the need to physically move to the hospital or to the physiotherapy office. In the future, medical doctors and physiotherapist could also propose some rehabilitation programs and check the efficacy and treatment compliance of a home-based rehabilitation program, as well as the improvements made by their patients through a connected interface (so to quickly adapt the training protocol).

Acknowledgments. This work was supported in part by funding by IDS company, TIMC-IMAG Laboratory, the French national program "programme d'Investissements d'Avenir IRT Nanoelec" ANR-10-AIRT-05, and Institut Universitaire de France.

\section{References}

1. Livre Blanc de la FESUM (1998), http://www.gem-sfcm.org .

2. Bach-y-Rita, P.: Sensory substitution and the human-machine interface. Trends Cogn. Sci.(Regul. Ed.) 7(12), 541--546 (2003).

3. Chenu, O., Vuillerme, N., Demongeot, J., Payan, Y.: A wireless lingual feedback device to reduce overpressures in seated posture: a feasibility study. PLoS One. 30;4(10):e7550 (2009).

4. Vuillerme, N., et al.: Sensory supplementation system based on electrotactile tongue biofeedback of head position for balance control. Neurosci Lett. 431(3), 206--210 (2008). 
5. Robineau, F., Boy, F., Orliaguet, J.P., Demongeot, J., Payan, Y.: Guiding the surgical gesture using an electro-tactile stimulus array on the tongue: a feasibility study. IEEE Trans Biomed Eng. 54(4):711-7 (2007).

6. Chenu, O., Vuillerme, N., Bucki M., Diot, B., Cannard, F. \& Payan Y. (2013). TexiCare: An innovative embedded device for pressure ulcer prevention. Preliminary results with a paraplegic volunteer. J Tissue Viability 22, 83--90 (2013).

7. Najeb, Y., Trafeh, M. : Résultats de la réparation primaire des plaies du nerf médian et du nerf ulnaire au poignet. Chir Main (2009).

8. Lenoble, E., Vilain, R.: Résultats de la réparation primaire de vingt- huit plaies isolées du nerf médian au poignet. Ann Chir Main 8(4), 347--51 (1989).

9. Witney, A., et al.: The cutaneous contribution to adaptive precision grip. Trends Neurosci 27(10), 637--643 (2004).

10. Dun, S., et al.: Lower median nerve block impairs precision grip. J Electromyogr Kinesiol 17(3), 348--354 (2007).

11. Schenker, M., et al.: Precision grip function after hand replantation and digital nerve injury. J Plast Reconstr Aesthet Surg 59(7), 706--716 (2006),

12. Li, Z.M., Nimbarte, A.D.: Peripheral median nerve block impairs precision pinch movement. Clin Neurophysiol, 117(9), 1941-8 (2006).

13. Monzée, J., et al. The effects of digital anesthesia on force control using a precision grip. $\mathbf{J}$ Neurophysiol 89(2), 672--83 (2003).

14. Augurelle, A.S., et al.: Importance of cutaneous feedback in maintaining a secure grip during manipulation of hand-held objects. J Neurophysiol, 89(2) 665--71 (2003)

15. Hermsdörfer, D.A., et al.: Grip force control during object manipulation in cerebral stroke. Clin Neurophysiol 114(5), 915--929 (2003).

16. Nowak, D.A., Hermsdörfer, D.A.: Selective deficits of grip force control during object manipulation in patients with reduced sensibility of the grasping digits. Neurosci Res, 47(1), 65--72 (2003).

17. Nowak, D.A., et al.: Moving objects with clumsy fingers: how predictive is grip force control in patients with impaired manual sensibility? Clin Neurophysiol 114(3), 472-487 (2003).

18. Bach-y Rita, P., et al. : Vision substitution by tactile image projection. Nature 221, 963-964 (1969)

19. Massimino, M.J.: Improved force perception through sensory substitution. Control Eng Pract 3(2), 215--222 (1995).

20. Visell, Y.: Tactile sensory substitution: Models for enaction in HCI. Interact Comput 21(1-2), 38-53 (2009)

21. Lederman, S.J., Browse, R.A.: The physiology and psychophysics of touch. Sensors and sensory systems for advanced robots. NATO ASI Series 43, 71-91 (1988).

22. Spicher, C.: Manuel de rééducation sensitive du corps humain. Médecine\&Hygiène, Geneva (2003).

23. Whitaker, T.A., et al. Vision and touch: Independent or integrated systems for the perception of texture? Brain Res 1242(C), 59--729 (2008).

24. Sourceforge : http://sourceforge.net/projects/zedgraph

25. Bell-Krotoski, J.: The repeteability of testing with Semmes-Weinstein monofilaments. J Hand Surg (1987). 